\title{
3 Research Square

\section{Agricultural Transfer Labor Wage Platform Based on Image Content Feature Retrieval and Internet of Things}

Ying Wu ( $\sim$ ying_wu0701@163.com )

Hubei Polytechnic Institute

Jikun Liu

University of Michigan

\section{Research Article}

Keywords: image content retrieval, artificial intelligence, agricultural transfer, labor, wage platform

Posted Date: January 21st, 2021

DOl: https://doi.org/10.21203/rs.3.rs-151212/v1

License: (c) (1) This work is licensed under a Creative Commons Attribution 4.0 International License.

Read Full License 


\title{
Agricultural transfer labor wage platform based on image
}

\section{content feature retrieval and Internet of Things}

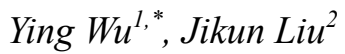 \\ ${ }^{1}$ Hubei Polytechnic Institute, Xiaogan, Hubei, 432000 \\ ${ }^{2}$ University of Michigan,Ann Arbor, 48104 \\ *Corresponding author: Ying Wu,Email:ying_wu0701@163.com
}

\begin{abstract}
Quick and accurate information identification of agricultural transfer labor wage platform is an essential function of labor intelligent management in the new era. Based on the content feature retrieval, this study constructs an artificial intelligence identity information recognition system and links the system to the salary platform. Simultaneously, this study uses the feature recognition to extract database information and realize intelligent salary assessment. In addition, the deep learning features used in this study are based on the positional information of the sift features and are finally calculated by the activation map to obtain a global vector of an image. Finally, this study design testing experiment to verify the performance of the algorithm. Through the output of the feature picture, it can be seen that the research algorithm has certain effects and can be used as a follow-up system practice.
\end{abstract}

Keywords: image content retrieval; artificial intelligence; agricultural transfer; labor; wage platform

\section{Introduction}

With the development of the economic level, the era of knowledge economy puts forward higher requirements for the management of wage information in enterprises and enterprises. By inputting the personnel salary file with a large amount of data and information into the computer and carrying out paperless management, not only the employee's salary file can be managed, but also the employee's business file and attendance file can be managed. Moreover, the above refined content has become an essential part of today's social production and work wage management ${ }^{[1]}$. Efficient and accurate personnel management can not only promote employees to continuously improve their own quality and work enthusiasm, but also improve the quality and efficiency of their work. With the rapid development of the information industry and Internet, the use of computers has penetrated into various fields ${ }^{[2]}$. This requires the computer to automate the processing of vast information, freeing managers from heavy manual labor to grasp information faster and more accurately. With the gradual maturity of computer science and technology, its powerful functions such as large amount of information storage, convenient operation, time saving, and reduced duplication of work have been deeply recognized by people and have played an increasingly important role in various fields of human social life and work ${ }^{[3]}$.

In order to adapt to the highly time concept of people in modern society, a "wage management system" was developed to improve the efficiency of enterprise management. While mitigating human labor, it enables people to get rid of the limitations of the original system. By clicking on the query, input, modification, output and other buttons on the computer, the above functions can be completed, which greatly reduces the working time and improves the work efficiency. The salary management system adopts the traditional Windows operation interface, and even non-computer professional personnel can operate skillfully in a short period of time. The overall task of system development is to enable office staff to complete the task of salary 
management quickly and easily ${ }^{[4]}$.

Since the end of the 20th century, the quietly informatized economy has rapidly penetrated into all aspects and fields of human society's life and work. It puts higher demands on human resource management and wage management that are increasingly increasing in the amount of data and information. Economic globalization is undergoing profound changes in the pattern of employee incentives, and wage and welfare design is more flexible and diversified. At the same time, the form of wage distribution has also shifted from a currency-led to a capital-led one ${ }^{[5]}$.

China's wage management system started in the mid-1970s. After more than 20 years of research and exploration, we now have a number of unique salary management systems, such as the Super Easy Personnel Valuation System, Zhuo Yan Salary Inquiry System, Ding Hengda B/S Personnel Salary System and so on. The initial salary management of enterprises and institutions was developed in languages such as Dbase III and Visual FoxPro ${ }^{[6]}$. Although these basic database programming languages can meet the needs of wage management at the time, the user interface design is not beautiful enough. Moreover, since its database technology uses a so-called "desktop database", data confidentiality is not strong, and it is not easy to maintain the security and integrity of large amounts of data.

In recent years, with the development and growth of domestic enterprises and institutions, the technical strength of the domestic wage management information system has been rapidly enhanced. At the same time, the domestic wage management information system has also turned to the real technology development stage. Some internationally advanced wage management techniques and concepts can be quickly applied in new management systems ${ }^{[7]}$. Moreover, many professional software manufacturers, the company's management system can closely follow the international advanced technology trends, reflecting a strong level of production and research and development. Nevertheless, how to further improve the technical research and development level of the wage management system and how to improve the management norms of the wage management system itself are still two problems currently faced by the wage management departments of enterprises and institutions in China. The domestic wage management information system is currently facing the peak period of upgrading the old management system to the new management system, and the defects and drawbacks of the traditional wage management methods and traditional wage management are being gradually improved ${ }^{[8]}$.

In foreign countries, the development of management information systems has been relatively early, and more advanced technologies such as cloud computing and big data have emerged. The management information system for wage management affairs is represented by software companies in developed countries such as the United States and Europe. Foreign products are generally integrated by multiple systems, and few systems for salary management are presented separately. They are composed of financial management system, decision support system, and daily affairs management system ${ }^{[9]}$. These systems are highly integrated, and each subsystem is managed collaboratively, data is shared, and at the same time independent of each other. In the process of system deployment and use, its management data is classified and entered, and the data of each module is completely shared, providing a comprehensive transaction management solution, integrating daily business management, salary management, financial management and decision management into one integrated management information system. At present, the development of foreign wage management information systems is mainly reflected in cloud computing, big data and multi-system integration, realizing the real-time sharing and 
intelligent processing of the system ${ }^{[10 \sim 12]}$. According to relevant statistics, more than $95 \%$ of the financial management transactions in the United States are processed by informatized computers, and the management of human resources, especially wage management transactions, has reached more than $90 \%$. The related system software of foreign wage management has great advantages in operation friendliness and comprehensiveness of functions ${ }^{[13]}$.

\section{Content-based image retrieval principle}

\subsection{Visual word bag model}

In the actual process, there is no feature that can perfectly represent the image content, and more retrieval errors are often generated under the interference of complex backgrounds. Therefore, if the image retrieval is performed only by image features, the application level cannot be achieved. Therefore, a carrier of image features and a data processing method of image features are required ${ }^{[14]}$.

For the processing of image features, the visual word bag inverted index model $(\mathrm{BoW})$ is a widely used method. Due to its outstanding simple structure, excellent performance and powerful expansion capabilities, it appears in practical applications at all levels. The specific steps are ${ }^{[15]}$ :

1) Some feature samples are randomly collected in a database with independent association, and a visual vocabulary codebook is trained offline by unsupervised clustering. The size of the visual vocabulary codebook is usually $10 \mathrm{k}, 20 \mathrm{k}, 100 \mathrm{k}, 200 \mathrm{k}$. According to actual needs, the most commonly used unsupervised clustering methods are standard k-means clustering, layered k-means, and approximate k-means.

2) For an image, the features in the image are first extracted, and then all the extracted feature quantities are transferred to the visual vocabulary codebook in step 1). At the same time, a series of image feature information is added to generate a BoW model, and the additional information may be location information of the feature, a binarization vector of the feature, and weight information of the data under a single vocabulary, as shown in Figure 1.

3) Based on the visual word bag in step 2), there are two ways to proceed with the image retrieval process in the next step. First, each image can be expressed as a histogram related to the visual vocabulary, and such a histogram is generated at the frequency of the features appearing in each visual vocabulary in the image, thereby calculating the similarity between the images. Second, using the voting mechanism, the weights are calculated for each feature in the image and accumulated to obtain image similarity.

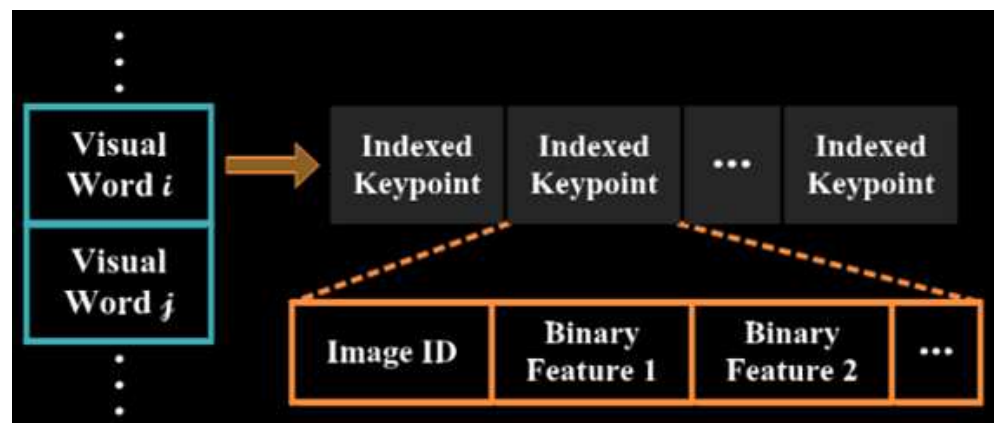

Figure 1 BoW model

\subsection{Voting mechanism based on BoW image retrieval}

This section will explain the voting mechanism in principle and clarify how the voting mechanism can be used to obtain the final search results in the BoW model.

There are two ways to get the BoW model to the final image retrieval result. The first type 
directly uses the frequency at which the features of the image appear on each visual vocabulary as the image representation, and then calculates the similarity. This method is simple, practical, and computationally fast, but the defect is fatal, and the retrieval accuracy is too low and there is basically no room for expansion. Therefore, this paper is more inclined to choose the second image retrieval process based on voting mechanism ${ }^{[16]}$.

For the image ${ }^{j=1 \mathrm{~L} n}$ in the database, the number of features in the image is represented by its local feature ${ }^{y_{i, j}}$. Then, the entire voting system can be boiled down to: Then, the entire voting system can be boiled down to:

1 The matching score of the image initialization similarity in the database can be defined as $s_{j}=0$.

2) For each feature ${ }^{x_{i}}$ of each query image and each feature ${ }^{y_{i, j}}$ of the image in the database, its similarity score ${ }^{s_{j}}$ will be updated as shown in equation (2):

$$
s_{j}:=s_{j}+f\left(x_{i}+y_{i, j}\right)
$$

In the formula, $\mathrm{f}$ denotes the matching function of the similarity between the responses $x_{i}$ and $y_{i, j}$.

Such a matching system is based on $\varepsilon-$ search and k-NN. Then, $f(.,$.$) , can be defined as$ equation (2):

$$
f_{\varepsilon}(x, y)=\left\{\begin{array}{c}
1 \text { if } d(x, y)<\varepsilon \\
0 \text { otherwise }
\end{array}\right.
$$

Or, equation (2)

$$
f_{k-N N}(x, y)=\left\{\begin{array}{c}
1 \text { if } f_{k-N N} \in x_{k-N N} \\
0 \text { otherwise }
\end{array}\right.
$$

In the formula, $d(.,$.$) represents the distance between two features in the feature space.$ (Usually, sift features use Euclidean distances to measure the distance between features).

3) Then, the final similarity score $s_{j}^{*}=g_{j}\left(s_{j}\right)$ of the image will be derived through a series of processes, such as equation (4):

$$
s_{j}^{*}=g_{j}\left(\sum_{i}^{m^{\prime}} \sum_{i=1}^{m_{j}} f\left(x_{i}, y_{i, j}\right)\right)
$$

In the formula, the simplest way to handle $g_{j}$ is $s_{j}^{*}=s_{j}$. In this case, the similarity score 
represents the number of registrations of the query image and the database image. It should be noted that the similarity score here may be calculated multiple times for one feature. Another well-known treatment is $s_{j}^{*}=s_{j} / m_{j}$. That is, the effect of the number of features of each image on the final result is considered, which reflects the proportion of matching features.

For the BoW model, the voting mechanism fits perfectly with its main steps. For ${ }^{x_{i}}$ and $y_{i, j}$, the quantification process q of the visual lexical codebook can be expressed as shown in equation (5) ${ }^{[16]}$ :

$$
\begin{aligned}
& q: x_{i} \rightarrow q\left(x_{i}\right) \\
& q: x_{x, y} \rightarrow q\left(x_{x, y}\right)
\end{aligned}
$$

Then, if there is $q\left(x_{i^{\prime}}\right)=q\left(x_{x, y}\right)$ in the same model, we can first judge that these two features belong to the same visual vocabulary. Furthermore, we can think that they match each other. The matching function can be defined as shown in equation (6):

$$
f_{q}\left(x_{i}, y_{i, j}\right)=\delta_{q\left(x_{i}\right), q\left(x_{x, y}\right)}
$$

In the formula, $\delta_{q(\cdot), q(.)}=1$. If two features are not assigned to the same visual vocabulary, $\mathrm{g}$ $\delta_{q(\cdot), q(\cdot)}=0$ exists. In addition, we can use BoW's good data processing capabilities to assign weight to each visual vocabulary. In general, the special processing method of idf is used, as shown in equation (7) ${ }^{[17]}$ :

$$
f_{\text {idf }}\left(x_{i}, y_{i, j}\right)=\left(i d f\left(q\left(x_{i}\right)\right)\right)^{2} \delta_{q\left(x_{i}\right), q\left(x_{x, y}\right)}
$$

Thus, after querying the image to complete the image of a database, the weight of each visual vocabulary can be obtained. Considering that there may be multiple features in each visual vocabulary from the query graph, it is necessary to take normalization $s_{j}^{*}=s_{j} / \sqrt{m_{j}}$. The reason for normalization using the $L_{2}$ norm is that this is closer to the actual feature matching ratio.

\section{Deep learning convolutional neural network mechanism}

Convolutional neural network is a kind of deep learning neural network, which has become a research hotspot in the field of image processing. The weight-sharing network structure makes it more suitable for human neural thinking, and also has a considerable reduction in complexity. Convolutional neural networks are highly invariant to translation, scaling, tilting, or common forms of deformation ${ }^{[18]}$.

The earliest CNN was produced in 1998 by LeNet, which is mainly used for the recognition of handwritten characters. After this, CNN has been silent for more than ten years. Until 2012, AlexNet made a blockbuster in the ImageNet Image Classification Contest. The reasons for success are nonlinear activation functions (ReLU), prevention of over-fitting (Dropout), big data training, and GPU applications:

1) ReLU activation function. The mathematical expression of ReLU is shown in equation (8): 


$$
f(x)=\max (0, x)
$$

Obviously, when the input signal is $<0$, the output is 0 . However, when the signal is $>0$, the output is equal to the input. ReLU only needs a threshold to get the activation value, instead of counting a lot of complex operations.

2) The principle of Dropout. All it does is set the output of each hidden layer neuron to zero with a probability of 0.5 . Because of this, networks need to be forced to learn more robust features that are useful when combined with some different random subsets of other neurons.Of course, its network design is also very skillful, the network structure shown in Figure 2. Since a single GPU has only $3 \mathrm{~GB}$ of memory, which limits the maximum size of its training network, the network is distributed across two GPUs.

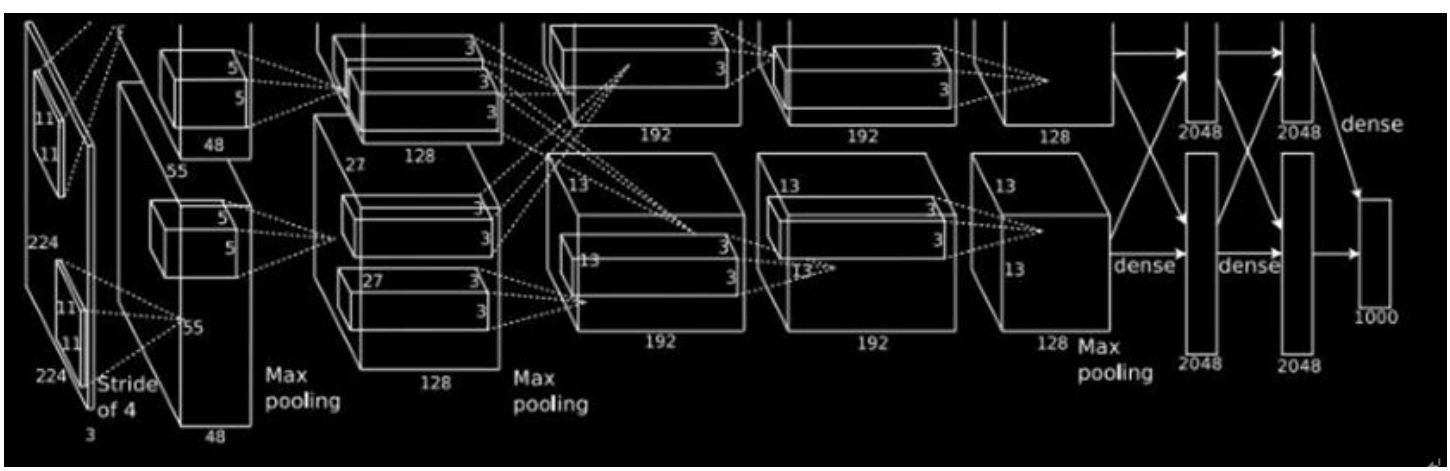

Figure 2 AlexNet architecture

Since the advent of AlexNet, the follow-up neural networks of other deep learning have mushroomed, but they are all similar. The result of processing each layer of convolutional layer is shown in Figure 3, and the changes in the timing of the use of ReLU and POOL are revealed.

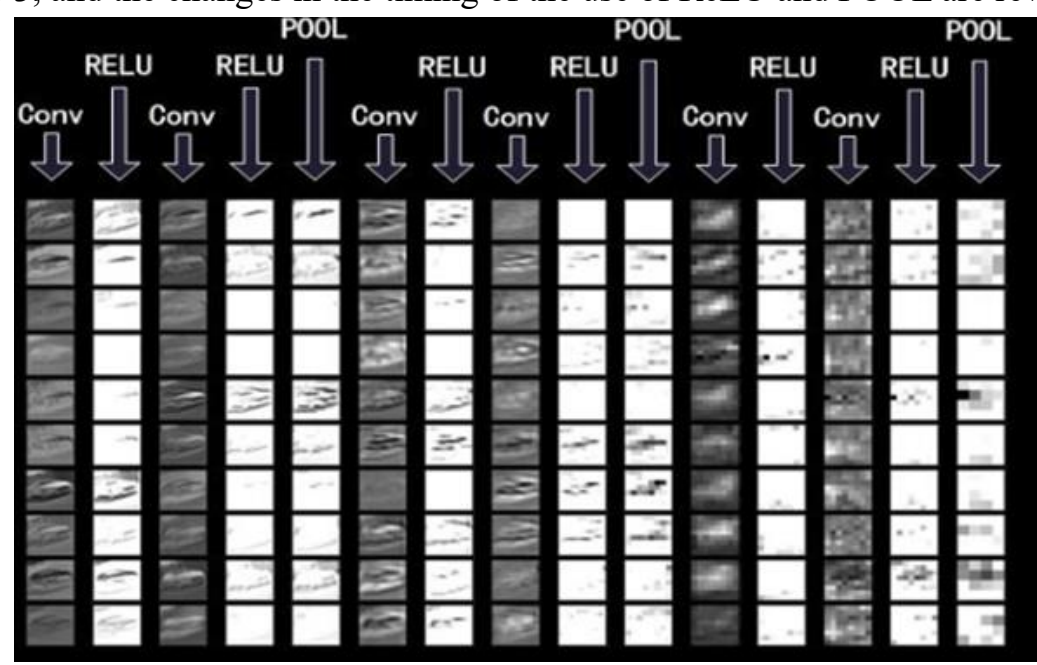

Figure 3 Schematic diagram of each layer of the convolutional neural network

Oxford Building Image Database (Oxford 5k): This database contains 5,062 images, provided by Flickr, and is divided into 11 categories. Each category is a combination of images from different viewpoints and illuminations at different scales, and each category has 5 query images. In order to ensure that the database has enough interference items, it also contains some people, objects and so on.

Paris Building Database (Paris 6k): This database contains 6412 images, provided by Flickr and divided into 11 categories. Each category is a combination of images from different 
viewpoints and illuminations at different scales, and each of them provides 5 query images. In order to ensure the interference of the database to the relevant images, many irrelevant images are doped.

\section{Fusion strategy of deep learning features and sift features}

In the process of convolutional neural network operation, images can obtain a series of features such as color, texture, and boundary. These features are structurally based on a single pixel of the image, which is closer to the original content and semantics in the global feature. The global feature has its advantages and there are no small defects._In order to improve the ability of global features to resist interference regions, this section intends to combine sift features with convolutional neural network features.

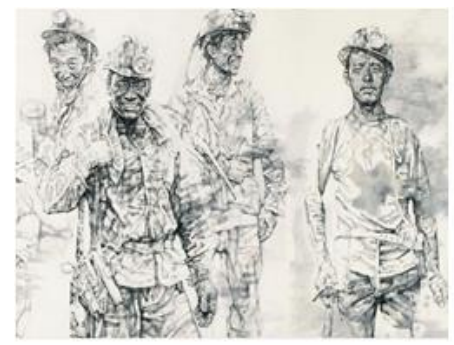

(a)

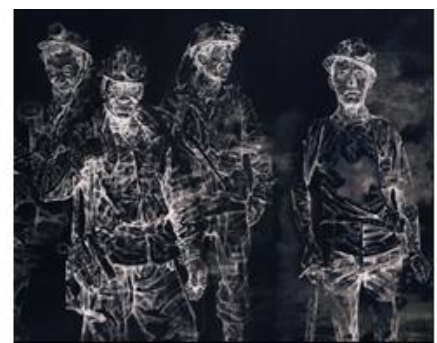

(b)

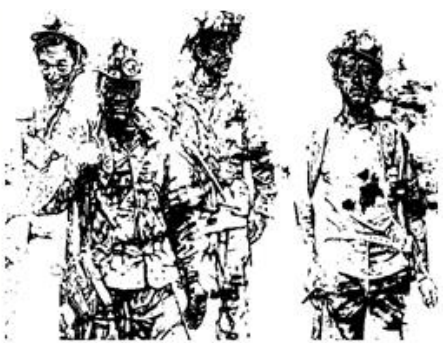

(c)

Figure 4 Features of the image (a) Original image (b) The distribution of sift features in the original image (c) Feature map of image convolutional neural network

The Sift features are based on image texture gradients and are mainly distributed in areas where the image content is complex, the texture is concentrated, the gradient span is large, and the pixel points are as shown in Figure 4(b). This description feature has a strong ability to express local details of the image, and the position in the image is also the most unique and representative position of each image. However, the convolutional neural network feature is an expression of a certain feature form of the whole image (such as: color, edge, texture, and different convolutional layer features). The result shown in Figure 4(c) is the result of the convolution layer from the last layer of the image in the VGG16 convolutional neural network model. Deep learning will become closer to the content and semantics of the image as the number of network layers increases. However, in general, if the number of layers is too deep, the trained network will have an over-fitting problem. Moreover, the global features will eventually contain many interference factors. In the past many algorithms, most of them have been fused and bridged by FV or VLAD. Moreover, the accompanying information at the time of sift feature extraction, that is, the position coordinates are not fully utilized. Therefore, the fusion strategy of sift features and deep learning features should be developed in conjunction with the location information of sift features and deep learning features.

The sift feature is extracted by the Hessian-Affine extractor and is provided by the image dataset. It should be noted that the extractor of the sift feature is not affected by the actual search results. The deep learning feature comes from the activation map obtained by the image in the 'pool5' layer of the VGG16 convolutional neural network model, and the activation map size is $1 / 32$ of the original image size. Figure 4(c) magnifies the picture for convenience of comparison.

We assume that the activation map of the image in the 'pool5' layer of the convolutional neural network is $X_{i, j, k} . \mathrm{i}, \mathrm{j}$ and k represent width (W), length (H), channel (C), respectively. We 
assume that the image sift feature group is $x_{z}$ and $p_{x_{z}}$ is the position coordinate information of the feature group $x_{z}$. Since the activation mapping of 'pool5' has been pooled 5 times, that is, the length and width are reduced to the original image, so if you need to deal with the correlation between the two, you need to adjust the size, as shown in formula (9).

$$
p_{x_{z}}^{\prime}=\operatorname{round}\left(\frac{p_{x_{z}}}{32}\right)
$$

We assume that $\alpha_{i, j}$ is a matrix with the same length and width cross-sectional dimensions as the activation map $X_{i, j, k}$. There are two ways to handle coordinates. One is to scale the coordinates in this chapter, and then generalize them to the nearest pixel on $\alpha_{i, j}$. The second is to perform equal-block and non-coincident seamless block division on the original image. The number of blocks should be the same as the number of ${ }^{\alpha_{i, j}}$ pixels, each block corresponds to a fixed pixel, and the feature points in the block are summarized to fixed pixels. The first method is simpler and faster than the second method, and it has no trouble in judging the block boundary. For the pixel points in $p_{x_{i}}^{\prime}$ that are summarized to $\alpha_{i, j}$, there are also two processing methods. One method is that it is simply represented by 0 or 1 , as shown in equation (10):

$$
a_{i, j}=\left\{\begin{array}{c}
1 \forall p_{x_{z}}^{\prime}=(i, j) \\
0 \text { otherwise }
\end{array}\right.
$$

Another method is to statistically summarize the number of pixels, as shown in equation (11):

$$
a_{i, j}=\left\{\begin{array}{c}
a_{i, j}+1 \text { if } p_{x_{z}}^{\prime}=(i, j), \mathrm{z}^{\prime} \in\{1,2, \mathrm{~L}, z\} \\
a_{i, j}+0 \text { otherwise }
\end{array}\right.
$$

For these two methods, since the number of sift feature points in each image is not the same and the difference is huge, if the second method is used, the similar images will be far apart, and the result will not rise and fall. Therefore, the first method of processing is preferred in practical method selection. Thus, the $\alpha_{i, j}$ is closer to the weight of $X_{i, j, k}$. Therefore, ${ }_{i, j, k}^{\prime}$ used as a representative image activation map is as shown in equation (12):

$$
X_{i, j, k}^{\prime}=\alpha_{i, j} X_{i, j, k}
$$

Then, due to the inconsistency of the sift points of each image, it is not suitable to use the accumulation pooling here. Therefore, this paper uses the maximum pooling to generate the expression $\mathrm{V}$ of the image, as shown in equation (13):

$$
V=\sum_{i=1}^{W} \sum_{j=1}^{H} x_{i, j, k}
$$




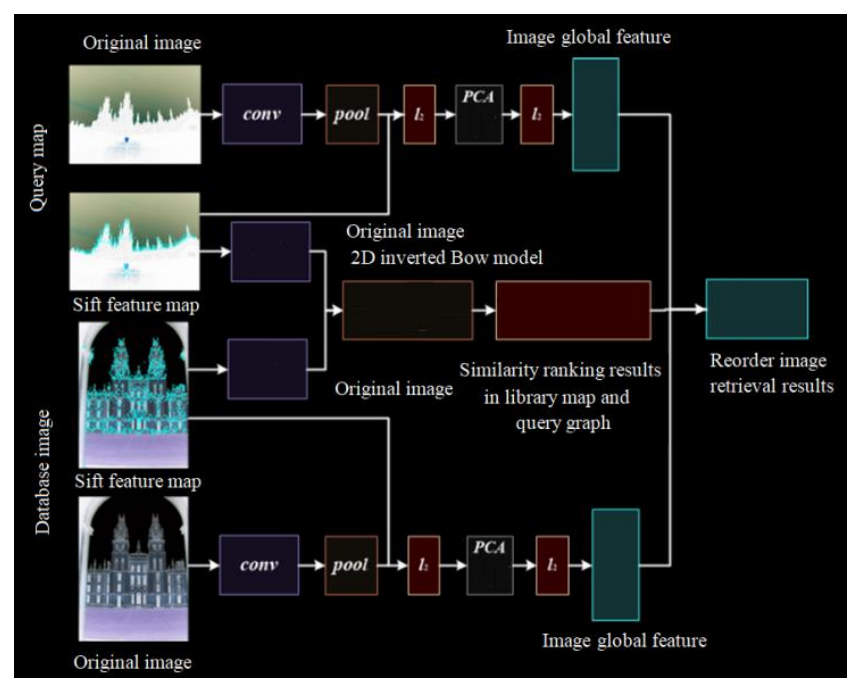

Figure 5 Flow chart of two-dimensional inverted index and deep learning feature image retrieval

The deep learning features used in this study were based on the location information of the sift features. Finally, by calculating the activation map, a global vector of an image is obtained. The two-dimensional inverted index model is a process of establishing a certain model by using two local features to perform a similar voting-like retrieval and sorting.

For the image $I_{z}$ that already has the similarity sequence in the image database, the specific reordering basis is the global feature of deep learning. The image representation $V_{r a f}(f \in[0, M])$ of the image of the first $\mathrm{M}$ number of the sequence $\mathrm{Ra}$ is selected, and the reordered reference vector $V_{r e}$ is obtained by the mean processing, as shown in equation (14):

$$
V_{r e}=\frac{\sum_{f=1}^{M} V_{r a f}}{M}
$$

In the formula, $V_{r e}$ is used to replace the original representation vector $\mathrm{V}$ of the image, and a general similarity measure and an image retrieval process are performed, as shown in Figure 5, thereby achieving correction and optimization of the two-dimensional inverted index image retrieval result. In the actual retrieval application, the image in the database has been extracted from the global feature extraction by the two-dimensional inverted index BoW model and global feature extraction based on CNN convolutional neural network.

\section{Agricultural transfer labor wage platform}

The server of the system is mainly composed of two database servers and one web server, and the directly connected network is a system network. For system users, they can access the system through the external network or intranet login account and perform related operations. The physical architecture design of the system is shown in Figure 6. 


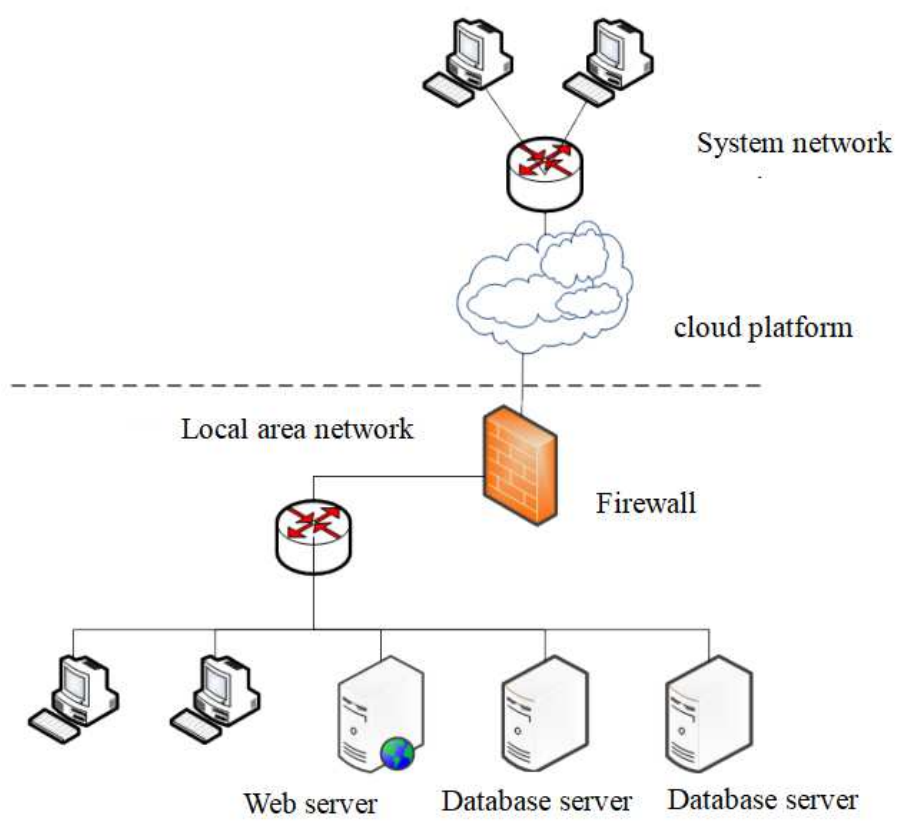

Figure 6 System physical architecture diagram

In the specific design implementation process, the related logic business package of personnel information management was designed. Through the logical business package, the related operations of the system personnel information management are completed, and the personnel information management business logic package is classified according to the basic data layer, the business logic layer and the presentation layer of the system, as shown in Figure 7 .

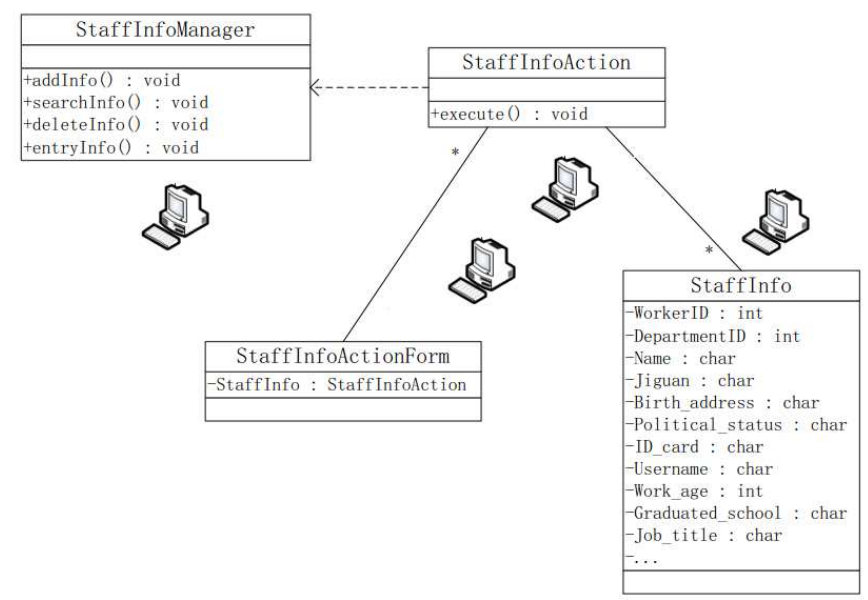

Figure 7 Class diagram of personnel information management

Next, a functional description of the functions of these classes is performed separately. StaffInfo Action class: Person information control class. It is mainly used to implement basic request for information addition, query, deletion, entry and other operations and page jump control. StaffInfo Manager class: Person information business logic class. It processes related business logic in the personnel information management, including logical processing operations such as adding, querying, deleting, and inputting personal information, which respectively correspond to the addInfo(), searchInfo(), deleteInfo(), and entryInfo() methods. StaffInfo class: Person information entity class. It mainly implements the encapsulation of personnel information, including the attributes of personnel information and methods for accessing these attributes, such 
as job number, department number, name, title and other attributes. StaffInfo ActionForm class: Person information form class. It is mainly used to encapsulate the details of the faculty staff information form and display it between pages.

For the security policy design of the system, it is necessary to set and support the system back-end database management software SQL Server. The management system uses Linux as the operating system running on the system server and uses SQL Server 2005 to store and manage all of the system's data. The security control strategy design of the system background database is shown in Figure 8.

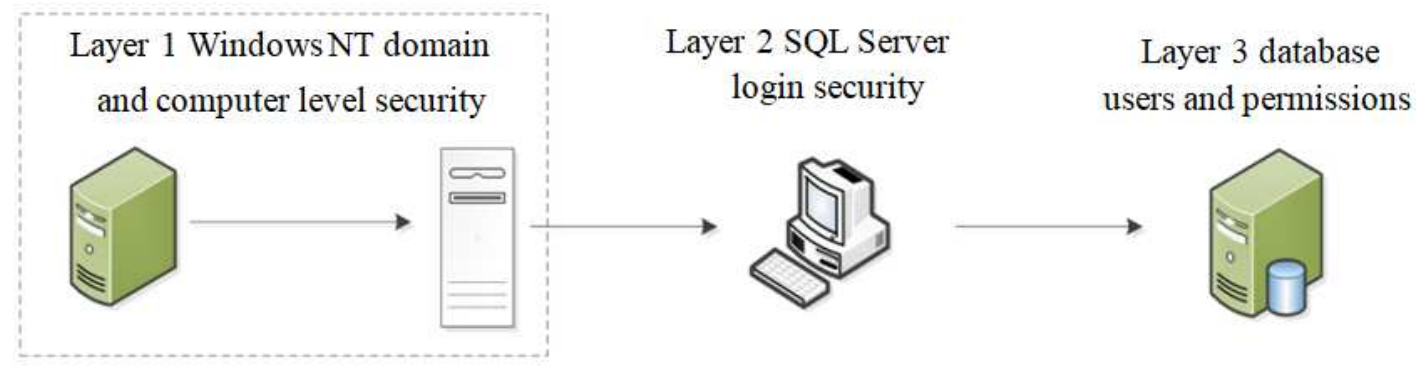

Figure 8 Schematic diagram of the security control strategy

As shown in the figure above, the security control policy setting of the system mainly utilizes the three-layer security policy of the SQL Server database. The first layer: the management interface of the SQL Server database in which the system administrator logs in to the system. SQL Server will map the user to a virtual NT account. Only after the user successfully passes the mapping login can he perform subsequent database management operations. Layer 2: It allows users to connect to a specific database; Layer 3: It allows users to have access to an object in the specified database.

\section{Analysis of image content retrieval performance}

On the basis of constructing the above salary platform, the system performance test is carried out, and the image content feature retrieval is mainly tested. The image content retrieval can realize the identity information of the migrant workers, the job recommendation and the salary assessment, and the system can be combined with the salary payment. The feature recognition effect is detected on the basis of constructing the research system, and the result is shown in Figure 9 .

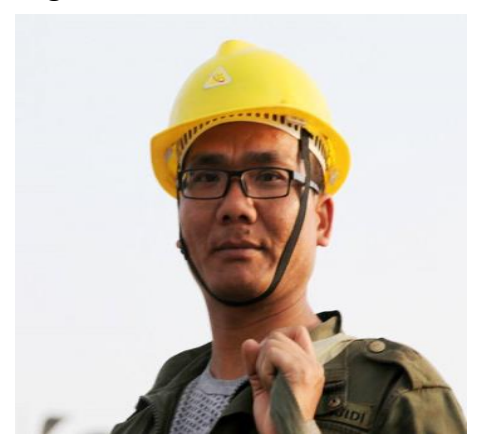

(a)

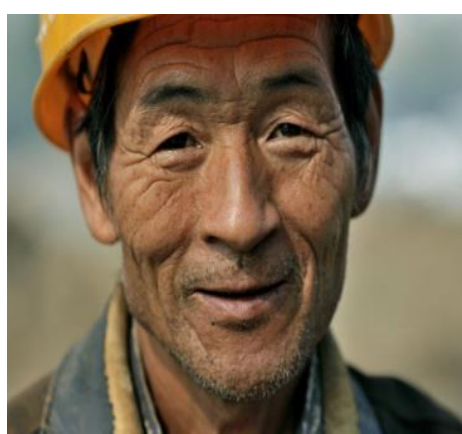

(b)

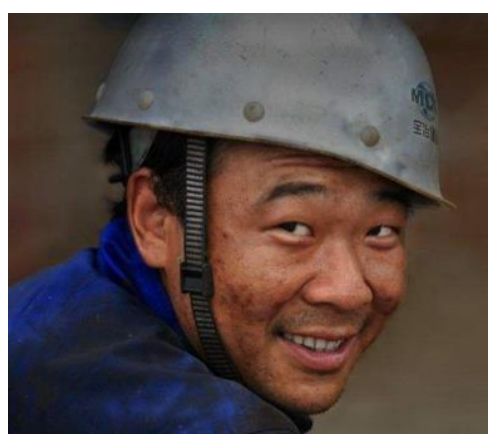

(c)

Figure 9 system identification case

After the system collects the corresponding image, it first performs preliminary processing on the image to retrieve the image features, and the obtained result is shown in Figure 10. 


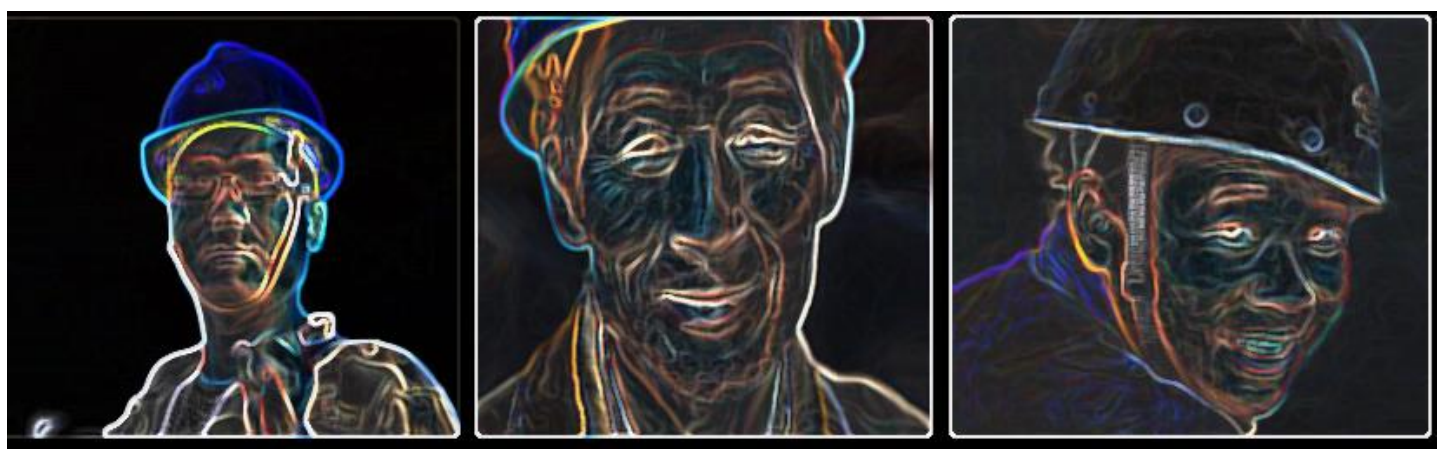

(a)

(b)

(c)

Figure 10 Preliminary image of image feature extraction

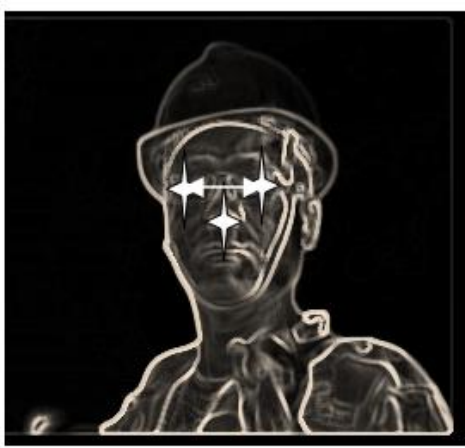

(a)

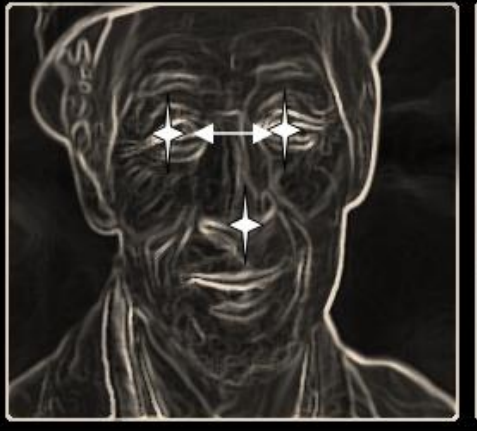

(b)

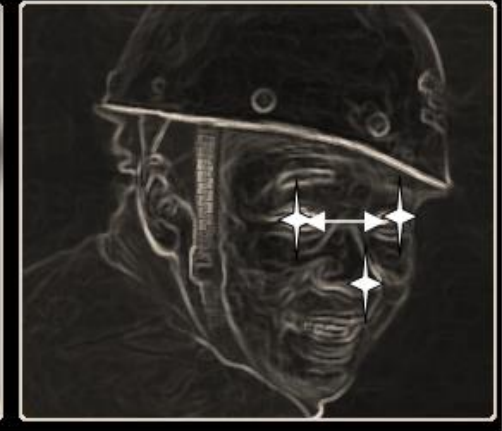

(c)

Figure 11 Feature extraction image

A further feature extraction image obtained on the basis of the preliminary extraction feature is shown in FIG. Through the recognition and judgment of pupil feature recognition, human facial features and skin color, it is possible to accurately determine the employee's corresf ond'ing code, collect the corresponding information from the database, and connect it to the agricultural transfer labor wage platform.

\section{Conclusion}

The development and use of the agricultural transfer labor wage platform is mainly carried out in the Web. When designing the system, it is necessary to consider the system network security problem and use the firewall to securely isolate the network. This design can prevent external attacks on the wage information management system and other information system related data information of the network operation, and effectively reduce the attack frequency of the external network to the system background server. The server of the system is mainly composed of two database servers and one web server, and the system network is directly connected. For system users, they can access the system through the external network or intranet login account and perform related operations. The deep learning feature used in this study is based on the position information of the sift feature, and finally the activation map is used to calculate the global vector of an image. The two-dimensional inverted index model is a process of establishing a certain model by using two local features to perform a similar voting-like retrieval and sorting. At the feature type level, the deep learning feature is a global feature, and the other is a model in which the local feature is established. Therefore, when the deep learning feature is referenced within the model or used as accompanying judgment information, there is no good insertion point or 
insertion condition. By constructing the system network and performing feature recognition verification, the algorithm and system of this research have certain practical effects.

\section{References}

[1] Kenthapadi K , Chudhary A , Ambler S . LinkedIn Salary: A System for Secure Collection and Presentation of Structured Compensation Insights to Job Seekers[J]. 2017.

[2] Beaumont B . Salary Systems in Public Higher Education[J]. Praeger, 2018.

[3] Shishkin S, Temnitsky A . From Salary to the Performance-Based Remuneration of Russian Physicians: How Motivation at Work is Changing[J]. HSE Working papers, 2017.

[4] Kenthapadi K , Ambler S , Zhang L, et al. Bringing Salary Transparency to the World: Computing Robust Compensation Insights via LinkedIn Salary[C]// the 2017 ACM. ACM, 2017.

[5] Manuel S. González Canché. Community College Scientists and Salary Gap: Navigating Socioeconomic and Academic Stratification in the U.S. Higher Education System[J]. Journal of Higher Education.

[6] Vaughan, Louella. The consultant on-call system is an extravagant waste of money[J]. BMJ, 2018:k2000.

[7] Eggleton K, Liaw W, Bazemore A. Impact of Gaps in Merit-Based Incentive Payment System Measures on Marginalized Populations[J]. The Annals of Family Medicine, 2017, 15(3):255-257.

[8] Liu D , Liang D . Three-way decisions in ordered decision system[J]. Knowledge-Based Systems, 2017:S0950705117304276.

[9] Guanxing X , Aimei L , Xiaotian W, et al. The Relationship between Salary Domain Discrepancy and Demission Decision:An Equate-to-Differentiate Theory Perspective[J]. Management Review, 2017.

[10] Chen, W. D . Upward wage rigidity and Japan $\mid$ "s dispatched worker system[J]. Economic Modelling, 2018:S0264999317318801.

[11] Gvetadze R S ， Butova V G ， Zuev M V , et al. [Regulatory framework of the new wage payment system in dentistry][J]. Stomatologiia, 2017, 96(5):14-18.

[12] Wright $\mathrm{C} \mathrm{H} \mathrm{G}$. Computational image processing for a computer vision system using biomimetic sensors and eigenspace object models[J]. Proceedings of SPIE - The International Society for Optical Engineering, 2017, 5299:327-336.

[13] Lefko C , Christi B , Tiffany E, et al. In Vitro Characterization of the Neurapheresis? System for the Treatment of Cryptococcal Meningitis[J]. Open Forum Infectious Diseases, 2017, 4(suppl_1):S481-S481.

[14] He X, Chen L, Li X, et al. Brain image feature recognition method for Alzheimer's disease[J]. Cluster Computing, 2018.

[15] Cui Y , Ma Y . Progress and Survey of Mobile Image Feature Recognition[J]. Recent Patents on Computer Science, 2017, 09(999):1-1.

[16] Sharan R V , Moir T J . Pseudo-color cochleagram image feature and sequential feature selection for robust acoustic event recognition[J]. Applied Acoustics, 2018, 140:198-204.

[17] Wang $Z$, Liu P , Cui T . RESEARCH ON FOREST FLAME RECOGNITION ALGORITHM BASED ON IMAGE FEATURE[J]. ISPRS - International Archives of the Photogrammetry, Remote Sensing and Spatial Information Sciences, 2017, XLII-2/W7:925-928. 
[18] Yan B , Tan W, Li K, et al. Codebook Guided Feature-Preserving for Recognition-Oriented Image Retargeting[J]. IEEE Transactions on Image Processing, 2017, 26(5):2454-2465. 


\section{Figures}

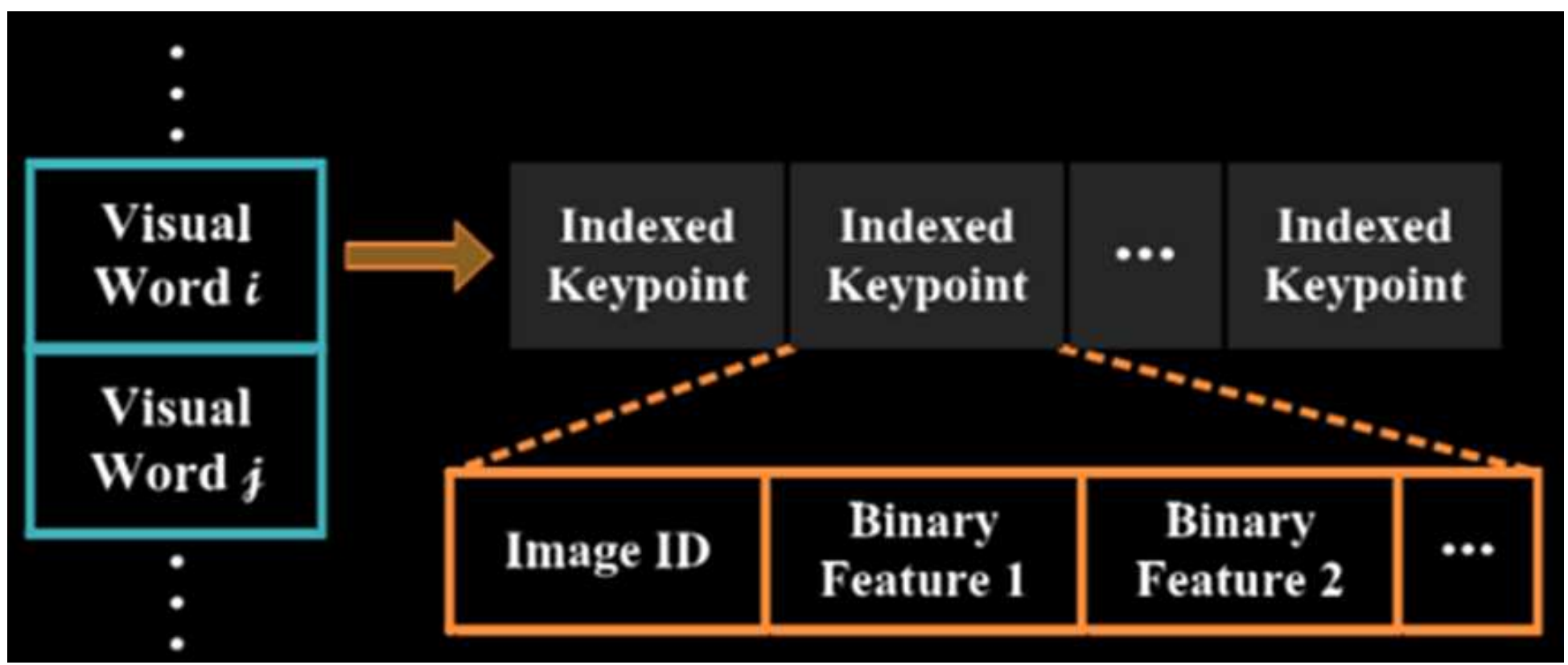

Figure 1

BoW model

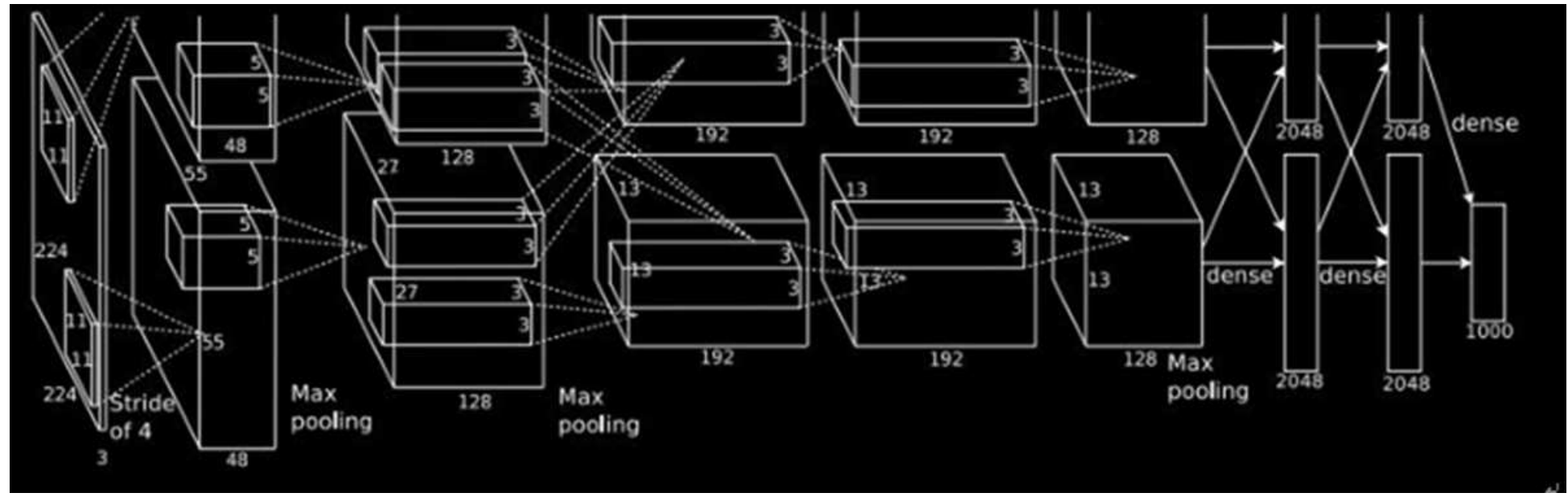

Figure 2

AlexNet architecture 


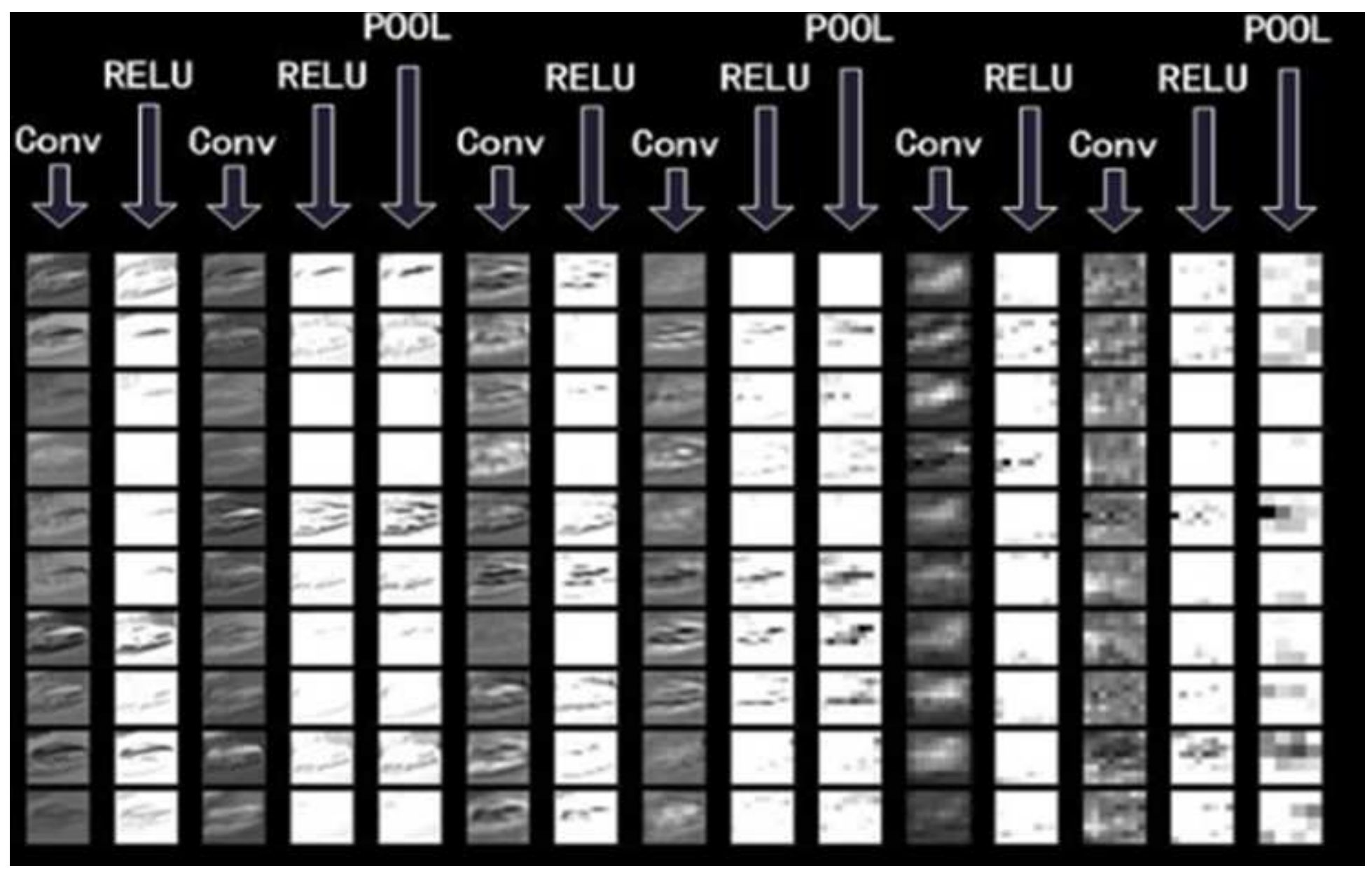

Figure 3

Schematic diagram of each layer of the convolutional neural network

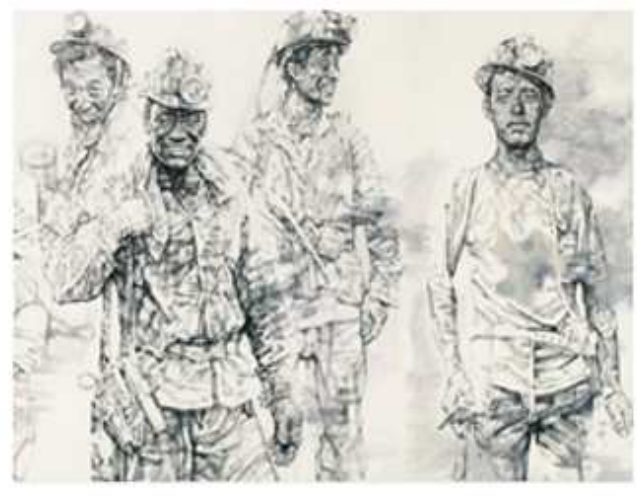

(a)

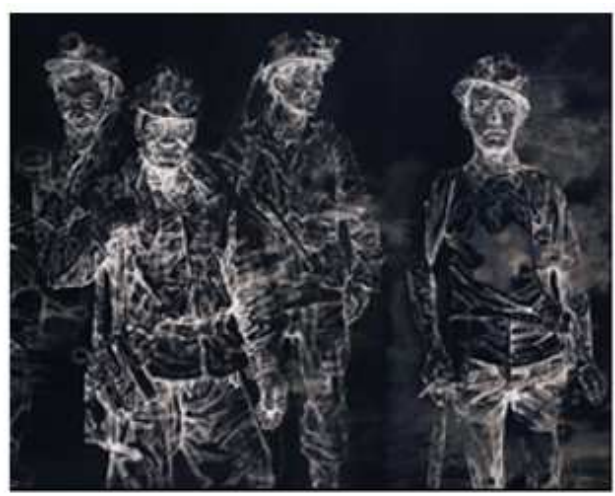

(b)

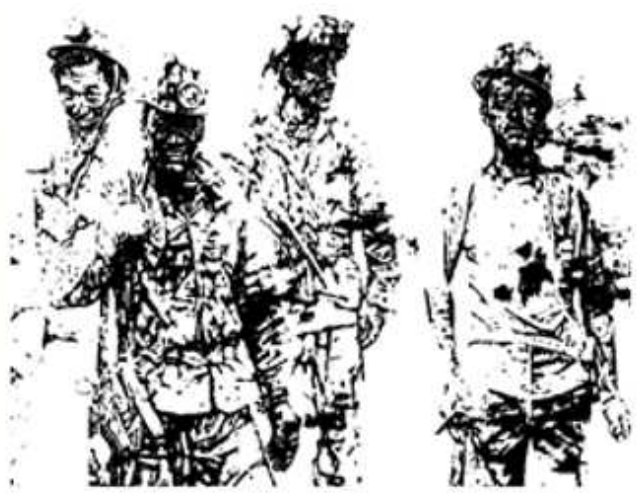

(c)

Figure 4

Features of the image (a) Original image (b) The distribution of sift features in the original image (c) Feature map of image convolutional neural network 


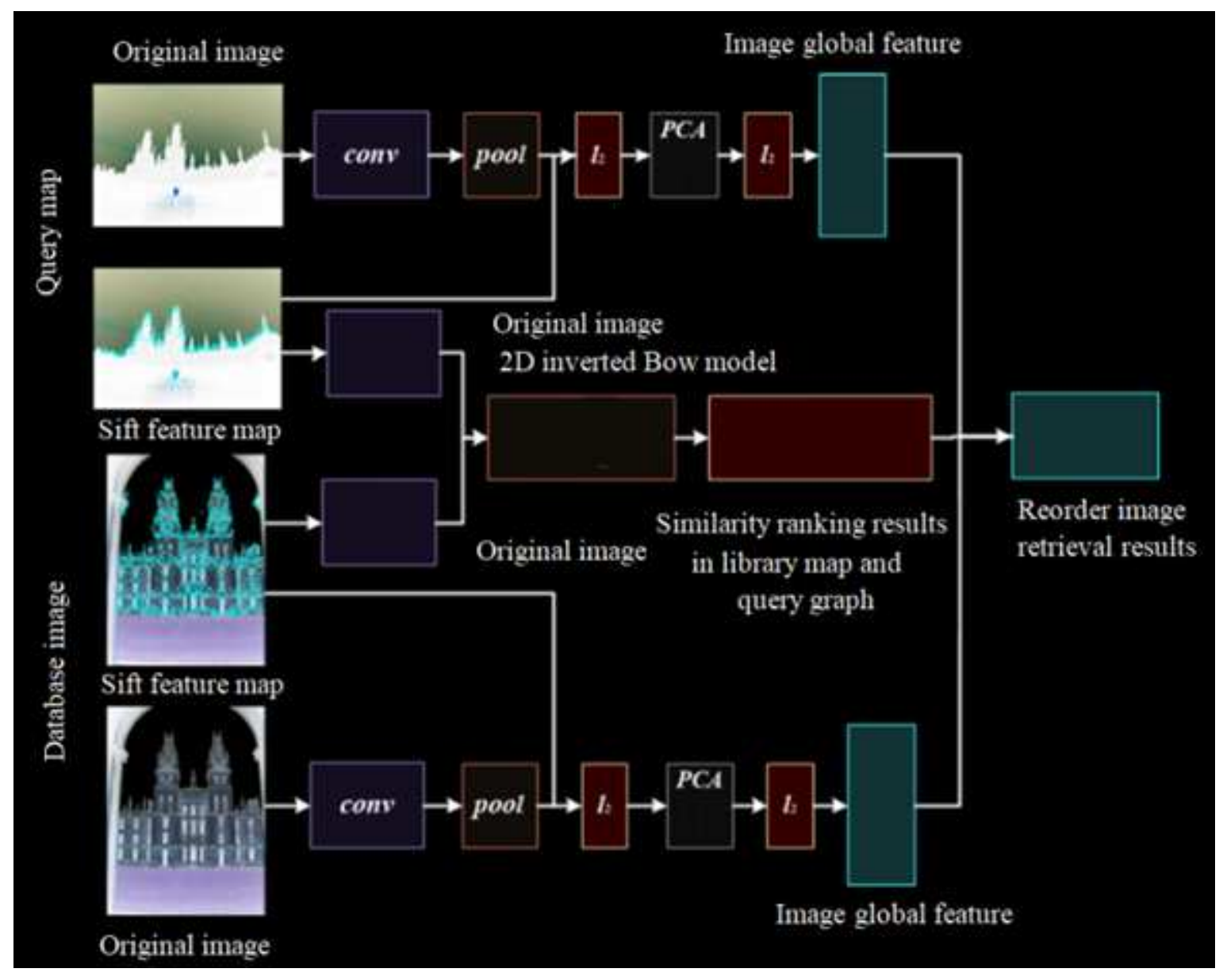

\section{Figure 5}

Flow chart of two-dimensional inverted index and deep learning feature image retrieval 


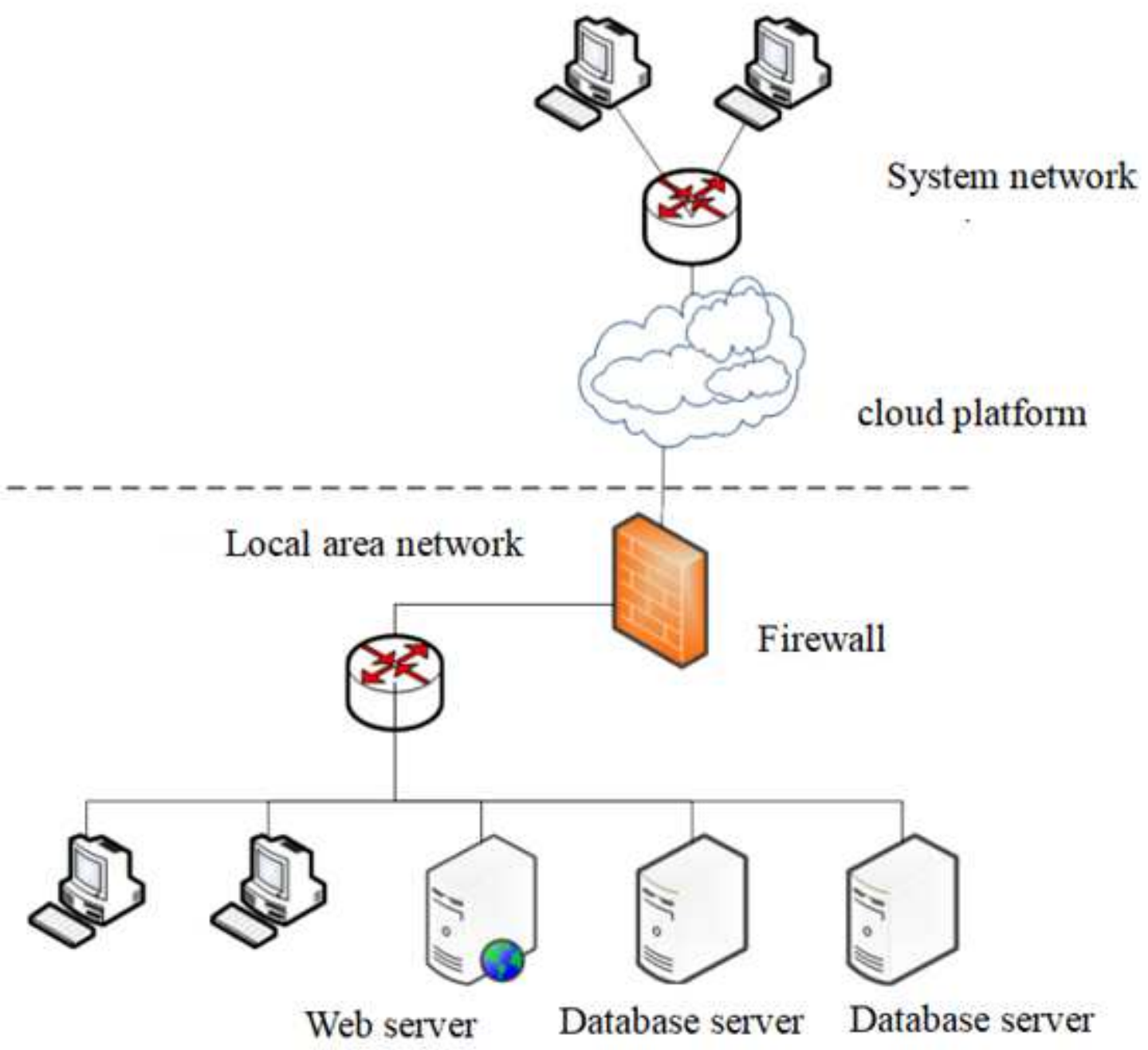

Figure 6

System physical architecture diagram

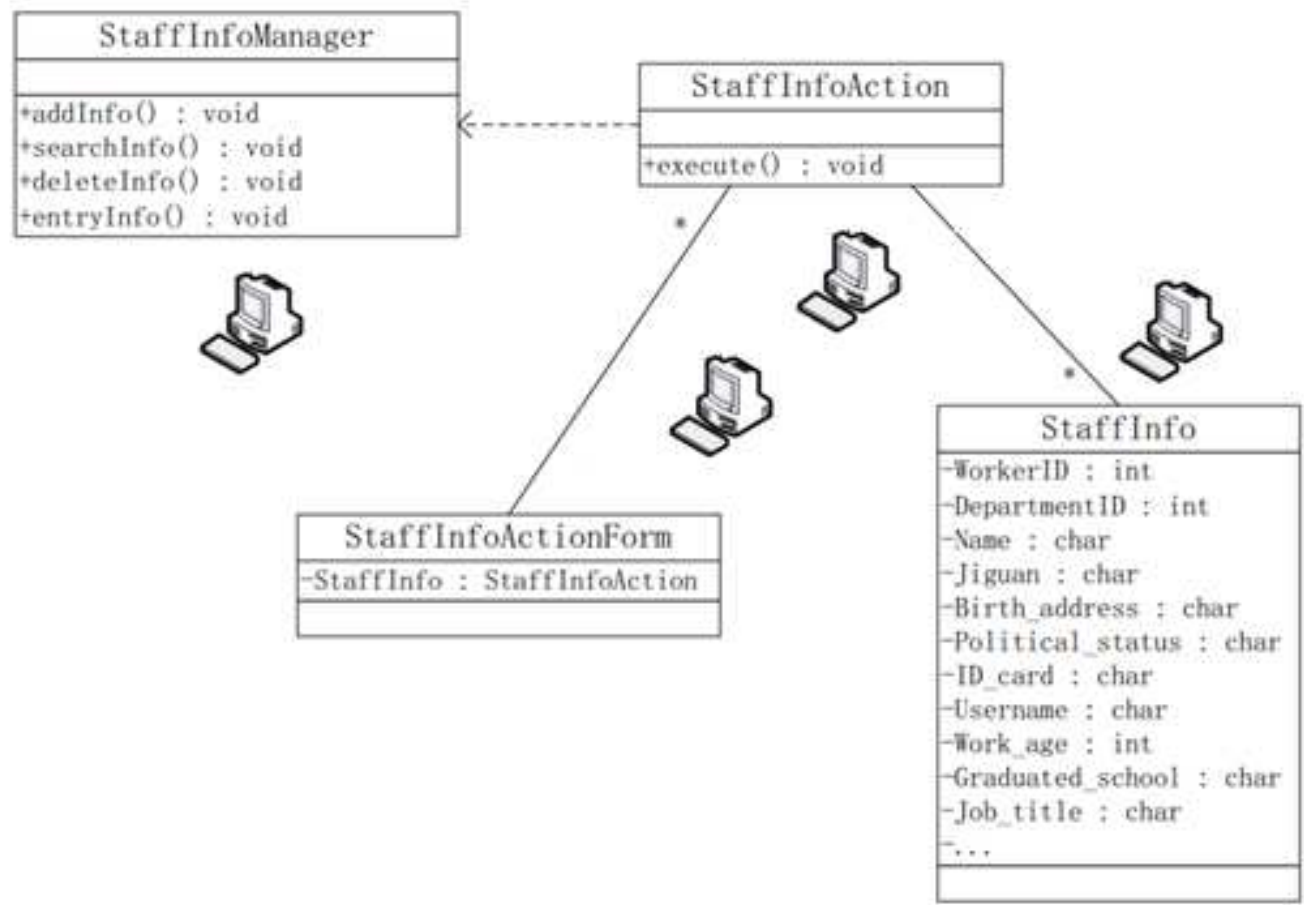


Figure 7

Class diagram of personnel information management

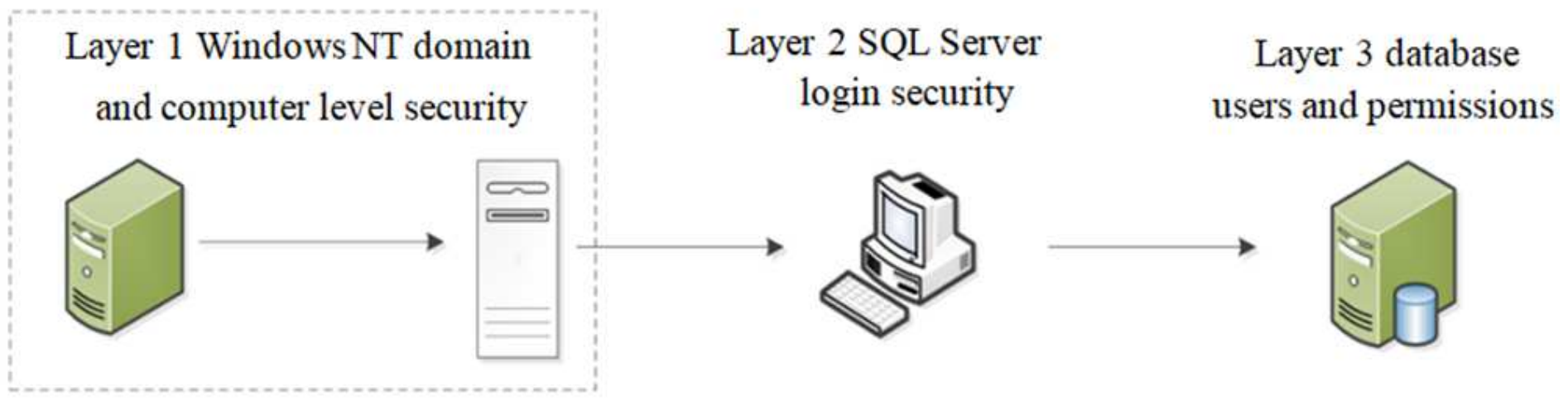

Figure 8

Schematic diagram of the security control strategy

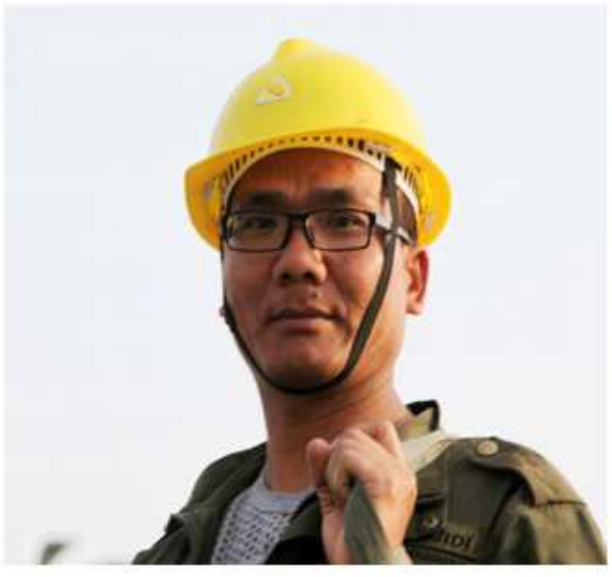

(a)

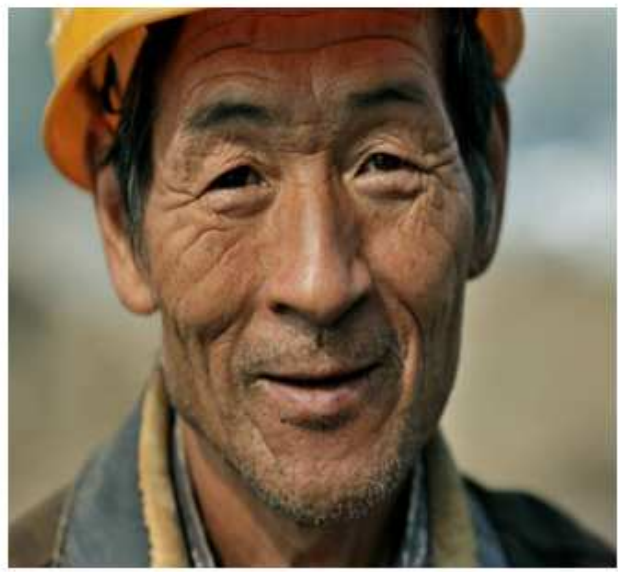

(b)

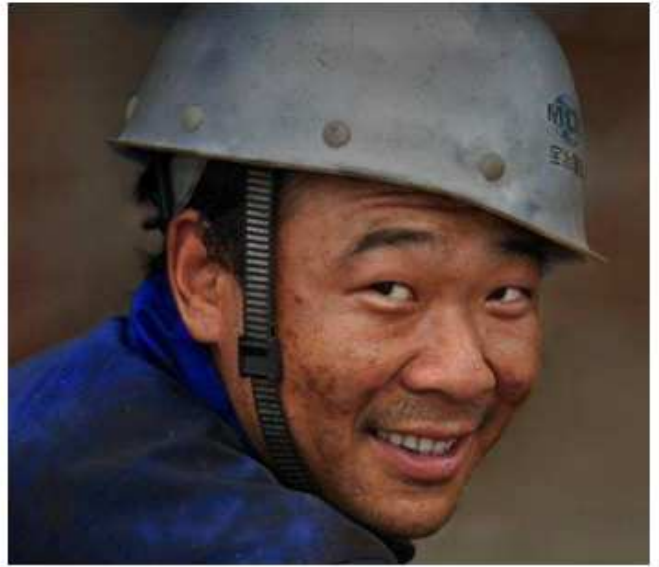

(c)

Figure 9

System identification case 


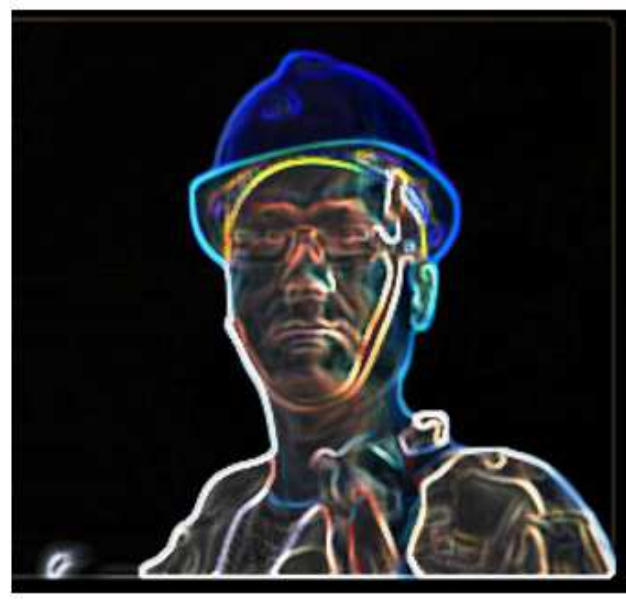

(a)

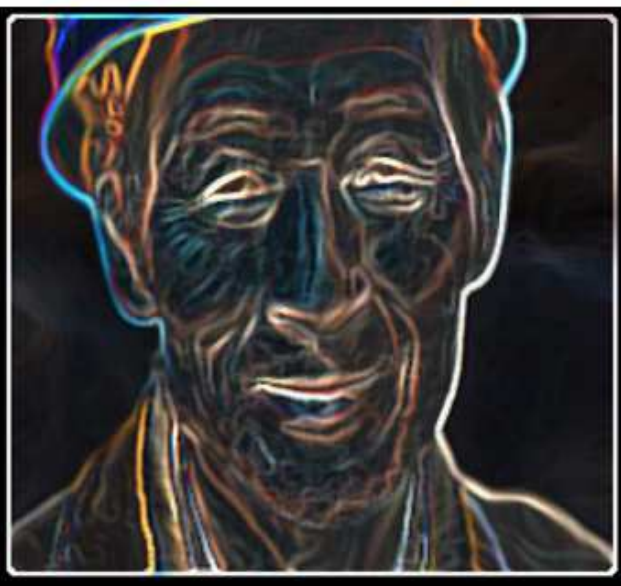

(b)

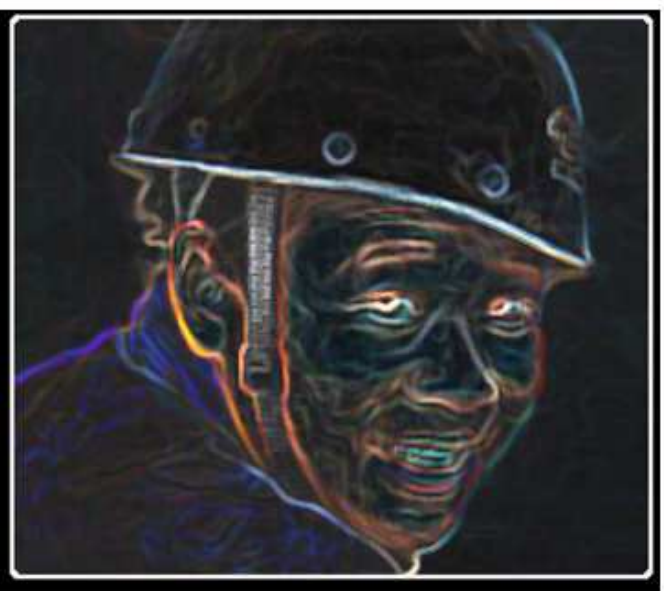

(c)

Figure 10

Preliminary image of image feature extraction

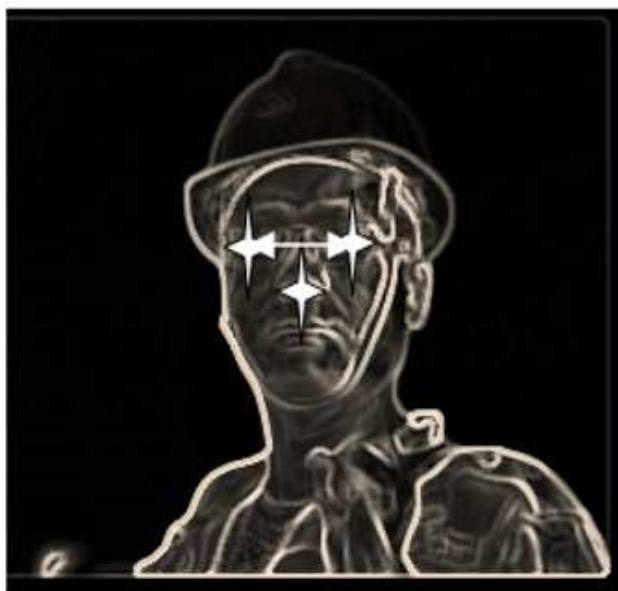

(a)

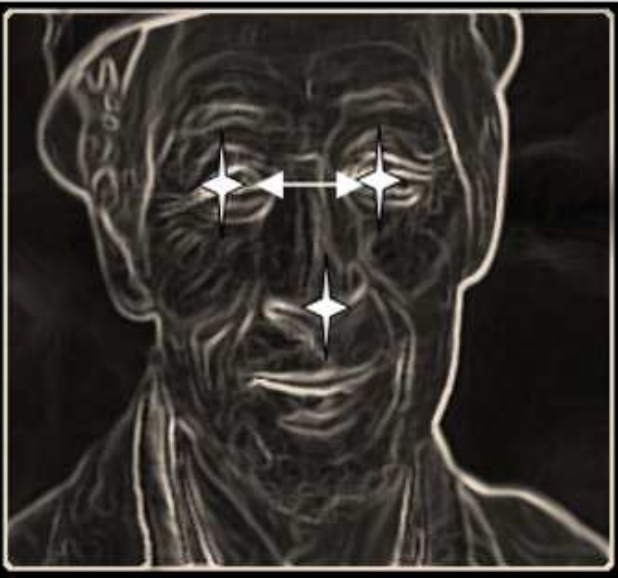

(b)

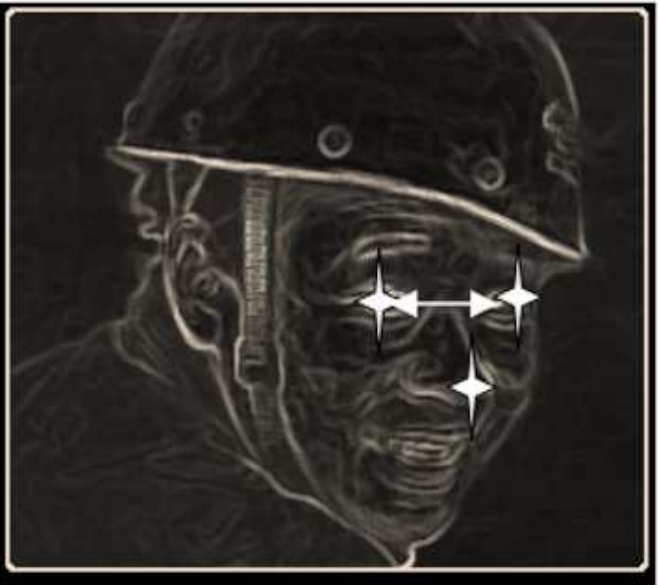

(c)

Figure 11

Feature extraction image 